\title{
Role of IncRNA BCYRN1 in trophoblast cell physiology and pathogenesis of preeclampsia
}

\author{
LIPING CHEN $^{1,2}$, QI SHI $^{3}$, BO FAN $^{3}$ and YAN CAI $^{1}$ \\ ${ }^{1}$ Antenatal Diagnosis Center, Affiliated Hospital of North Sichuan Medical College; ${ }^{2}$ School of Clinical Medicine, \\ North Sichuan Medical College; ${ }^{3}$ Department of Gynecology and Obstetrics, \\ Affiliated Hospital of North Sichuan Medical College, Nanchong, Sichuan 637000, P.R. China
}

Received September 21, 2020; Accepted April 12, 2021

DOI: $10.3892 /$ etm.2021.10571

\begin{abstract}
Long non-coding RNAs (lncRNAs) may play a key role in the pathogenesis of preeclampsia (PE). The present study investigated the role of the lncRNA brain cytoplasmic RNA 1 (BCYRN1) in PE. A total of 30 patients with severe PE (SPE) and 30 patients with mild PE (MPE) were recruited, whilst 30 healthy pregnant individuals were enrolled as controls. Placental tissues of enrolled subjects were collected after delivery. The clinical data of pregnant women and newborns were recorded before the correlation between BCYRN1 expression and clinical characteristics was analyzed. Furthermore, HTR-8/SVneo cells were transfected with BCYRN1 overexpression plasmids and BCYRN1 small interfering (si)RNA. Cell Counting Kit-8, Transwell, flow cytometry and tube formation assays were used to detect the function of BCYRN1 in HTR-8/SVneo cells. Reverse transcription-quantitative PCR was used to detect BCYRN1 expression in placental tissues and HTR-8/SVneo cells. Western blotting was used to detect the protein expression levels of Wnt1 and $\beta$-catenin. BCYRN1 expression was lower in placenta with mild PE compared with in normal placenta, and was in turn lower in placenta with severe PE. BCYRN1 was negatively correlated with systolic blood pressure and 24-h urinary protein in patients with PE. BCYRN1 siRNA inhibited cell viability, migration, invasion and tube forming abilities whilst increasing apoptosis. By contrast, BCYRN1 overexpression conferred opposite effects. The levels of Wnt1 and $\beta$-catenin expression in the cells and placental tissues were next measured. Cells overexpressing BCYRN1 were further treated with the Wnt pathway inhibitor XAV939. Wnt1 and $\beta$-catenin expression were elevated when BCYRN1 was
\end{abstract}

Correspondence to: Dr Yan Cai, Antenatal Diagnosis Center, Affiliated Hospital of North Sichuan Medical College, 63 Wenhua Road, Nanchong, Sichuan 637000, P.R. China E-mail: drcaiyan0702@163.com

Key words: preeclampsia, long non-coding RNA brain cytoplasmic RNA 1, Wnt/ $\beta$-catenin pathway, placenta, trophoblasts, invasion, apoptosis overexpressed, but were decreased after BCYRN1 knockdown. XAV939 attenuated the effect of BCYRN1 overexpression on HTR-8/SVneo cells. Overall, the resulted indicated that upregulation of BCYRN1 increased trophoblast viability and prevented apoptosis by activating the $\mathrm{Wnt} / \beta$-catenin pathway to delay PE onset.

\section{Introduction}

Preeclampsia (PE) is a predominant pregnancy-specific vascular disorder of increasing incidence, which is also a major cause of maternal and infant morbidity and mortality (1). PE affects $5-7 \%$ of all pregnant women and is responsible for $>70,000$ maternal and 500,000 fetal mortality worldwide every year (2). PE is characterized by new-onset hypertension and proteinuria after 20 weeks of pregnancy (3). Individuals with PE and their children are at a higher risk of developing severe cardiovascular complications and metabolic disorders (4). Clinical and pathological studies previously confirmed that placental dysplasia, excessive maternal inflammation and endothelial dysfunction are central to the pathogenesis of PE $(2,5)$. Accumulating evidence has also shown that the etiology of PE involves poor placentation due to inadequate trophoblast invasion $(6,7)$. Therefore, it is imperative to perform preclinical experiments in trophoblasts to elucidate the mechanism underlying PE pathogenesis.

It has been previously indicated that long non-coding RNAs (lncRNAs) can serve active roles in numerous cellular processes, including regulation of gene expression, and post-transcriptional and epigenetic modifications in the development of most organs, such as the placenta $(8,9)$. Genome-wide lncRNA expression profile studies in PE placentas using microarray have highlighted a number of differentially expressed lncRNAs in placentas with PE compared with in normal placenta $(10,11)$. In particular, lncRNAs, including H19, metastasis associated lung adenocarcinoma transcript 1 and HOX antisense intergenic RNA, have all been reported to regulate the pathogenesis of PE (12-14). An existing review outlined that lncR NAs have an impact on both the occurrence and development of PE through changes in the biological functions of trophoblasts, immune regulation, epigenetic regulation and energy 
metabolism (15). The aim of the present study was to identify new lncRNAs to explore the potential mechanism of PE. Brain cytoplasmic RNA 1 (BCYRN1) is a brain-specific lncRNA with a confirmed regulatory role in dendritic translation in neurons (16). It has also been reported that BCYRN1 is expressed in a variety of cancer cells, such as colon cancer and esophageal cancer cells, which can regulate the expression of various proteolytic enzymes and promote tumor migration and invasion $(17,18)$. Since BCYRN1 can promote the proliferation and migration of airway smooth muscle cells $(19,20)$, it may also affect the biological function of non-cancer cells. Trophoblasts have been recognized to share histological and behavioral characteristics with cancer cells (21). It has been widely reported that insufficient trophoblast infiltration is a key cause of PE (22). As such, the expression and activation of proteolytic enzymes is required for the invasion of ectotrophoblast cells into the decidua and subsequent initiation of vascular remodeling $(23,24)$. However, whether lncRNA BCYRN1 can exert regulatory effects on trophoblast cells warrants further investigation. Therefore, the present study aimed to identify the expression profile and clinical relevance of the IncRNA BCYRN1 in placentas with PE, to explore the effect of lncRNA BCYRN1 on the biological physiology of HTR-8/SVneo trophoblasts and their possible underlying mechanism.

\section{Materials and methods}

Study subjects. A total of 30 patients with mild PE (MPE group) and 30 patients with severe PE (SPE group) who underwent cesarean section at the Affiliated Hospital of North Sichuan Medical College (Nanchong, China) between April 2017 and April 2019 were recruited. In addition, 30 healthy pregnant women who delivered via cesarean section were selected as the control group (Normal group). All pregnancies were singleton and delivered via cesarean section. Those with pregnancy-related or other complications, including gestational diabetes mellitus, primary hypertension and chronic nephritis, were excluded. The diagnostic criteria of SPE according to the 8th edition of Obstetrics and Gynecology published by the People's Medical Publishing House were used (25). The specific diagnostic criteria for PE were as follows: i) $\geq 2$ blood pressure rises [systolic blood pressure (SBP) $\geq 140 \mathrm{mmHg}$ or diastolic blood pressure (DBP) $\geq 90 \mathrm{mmHg}$ ] after 20 weeks of gestation at an interval time of $\geq 6 \mathrm{~h}$; and ii) 24-h urine protein $\geq 300 \mathrm{mg}$. Patients with PE who met $\geq 1$ of the following criteria were diagnosed as SPE: i) SBP $\geq 60 \mathrm{mmHg}$ or DBP $\geq 110 \mathrm{mmHg}$; ii) platelet count $<100 \times 10^{9} / 1$; iii) liver function damage (serum transaminase concentration $>2$ times of the upper limit of the normal range ( $\sim 0-40 \mathrm{U} / \mathrm{l}))$; iv) renal function impairment, such as the $24-\mathrm{h}$ urine protein $\geq 2 \mathrm{~g}, 24$-h urine volume $<400 \mathrm{ml}$ or serum creatinine $>106 \mu \mathrm{mol} / \mathrm{l}$; v) pulmonary edema; vi) persistent headache, visual impairment or other abnormalities of central nervous system; vii) heart failure; viii) fetal growth restriction or oligohydramnios and placental abruption; and ix) intravascular hemolysis, including anemia, jaundice and increased lactate dehydrogenase levels. Patients who were not diagnosed with SPE were considered MPE. The present study was approved by the Academic Ethics Committee of the Affiliated Hospital of North Sichuan
Medical College and informed consent was obtained from all individuals.

Sample collection. Placental tissues of the enrolled individuals were obtained $15 \mathrm{~min}$ after delivery. Placental biopsies $(2 \times 2 \times 2 \mathrm{~cm})$ were collected at $3,6,8$ and 12 o'clock on the maternal surface and at the central position to avoid the placental margin, calcification, hemorrhage and necrosis. The placental tissues were washed with PBS, frozen in liquid nitrogen and stored at $-80^{\circ} \mathrm{C}$ for $\mathrm{mRNA}$ and protein extraction after the blood in the tissue was removed with sterile filter paper. The diagnosis of preeclampsia and grading in this study was not based on pathological tissues, but on clinical symptoms and indicators. Therefore, hematoxylin and eosin staining was not performed on the placental tissues.

Cell culture. HTR-8/SVneo cells, (American Type Culture Collection), which are frequently used to study the function of trophoblasts, were generated by using freshly isolated extravillous cytotrophoblasts from first trimester placenta and transfected with a plasmid containing the simian virus 40 large $\mathrm{T}$ antigen (26). It contains two populations, one of epithelial and one of mesenchymal origin (27). HTR-8/SVneo cells were seeded in a $25-\mathrm{cm}^{2}$ flask and cultured in RPMI-1640 (Thermo Fisher Scientific Inc.) containing 10\% fetal bovine serum (FBS; PAN-Biotech $\mathrm{GmbH}$ ), $100 \mathrm{U} / \mathrm{ml}$ streptomycin and $100 \mathrm{U} / \mathrm{ml}$ penicillin at $37^{\circ} \mathrm{C}$ and $5 \% \mathrm{CO}_{2}$. After the HTR-8/SVneo cells grew to $\sim 75 \%$ confluency, the cells were detached with $1 \mathrm{ml}$ $0.25 \%$ trypsin (Sinopharm Chemical Reagent Co., Ltd.) and passaged at a ratio of 1:2. The cells in the logarithmic growth phase were selected for subsequent experiments.

Cell transfection. Sequences for small interfering (si)RNAs against BCYRN1 (forward, 5'-UUGCUUUGAGGGAAGUUA C-3' and reverse, 5'-GUAACUUCCCUCAAAGCAATT-3'), negative control (NC) siRNA (forward, 5'-UUCUCCGAA CGUGUCACGU-3' and reverse, 5'-ACGUGACACGUUCGG AGAA-3'), the recombinant plasmid pcDNA3.1-BCYRN1 and pcDNA3.1 empty vector were purchased from Shanghai GenePharma Co., Ltd. HTR-8/SVneo cells were placed in 96-well plates $\left(5 \times 10^{3}\right.$ cells/well) and transfected using Lipofectamine $^{\circledR} 3000$ (Thermo Fisher Scientific, Inc.) when the cells reached $70-90 \%$ confluence. Cells were assigned into the si-BCYRN1 $(2 \mu \mathrm{l})$, si-NC, pcDNA3.1-BCYRN1 $(1,000 \mathrm{ng})$ and pcDNA3.1 groups. At $12 \mathrm{~h}$ post-siRNA-transfection or $8 \mathrm{~h}$ post-vector-transfection, the medium was refreshed and cells were cultured further at $37^{\circ} \mathrm{C}$ with $5 \% \mathrm{CO}_{2}$. Untransfected cells were used as the control group. Total RNA was extracted at $48 \mathrm{~h}$ post-transfection and BCYRN1 expression was verified via reverse transcription quantitative polymerase chain reaction (RT-qPCR). In addition, HTR-8/SVneo cells transfected with pcDNA3.1-BCYRN1 for $24 \mathrm{~h}$ were incubated in the medium containing the Wnt signaling inhibitor XAV939 (2 $\mu \mathrm{mol} / \mathrm{l}$; cat. no. HY-15147; MedChemExpress) at $37^{\circ} \mathrm{C}$ for $24 \mathrm{~h}$, whilst the medium supplemented with dimethyl sulfoxide (DMSO) was used as a control.

Cell Counting Kit-8 (CCK-8) assay. HTR-8/SVneo cells were plated into 96 -well plates at 5,000 cells/well and placed in $5 \% \mathrm{CO}_{2}$ at $37^{\circ} \mathrm{C}$. In total, $20 \mu \mathrm{l} \mathrm{CCK}-8$ (Nanjing Jiancheng 
Table I. Primer sequences used for reverse transcription-quantitative PCR.

\begin{tabular}{lll}
\hline Gene & \multicolumn{1}{c}{ Forward, 5'-3' } & \multicolumn{1}{c}{ Reverse, 5'-3' } \\
\hline lncRNA BCYRN1 & TCAGAGCGACAATTTGAGATC & GCAGTAGCAGCAGCATTTC \\
GAPDH & GATTGTTGCCATCAACGACC & GTGCAGGATGCATTGCTGAC
\end{tabular}

lncRNA, long non-coding RNA; BCYRN1, brain cytoplasmic RNA 1.

Bioengineering Institute) detection solution was added to each well at $37^{\circ} \mathrm{C}$ for $1 \mathrm{~h}$. Absorbance values at $450 \mathrm{~nm}$ were detected in each well at $0,24,48,72$ and $96 \mathrm{~h}$, respectively, using an automatic microplate reader (Bio-Rad 680; Bio-Rad Laboratories, Inc.).

Flow cytometry. The original culture medium of HTR-8/SVneo cells was first discarded before $1.5 \mathrm{ml} 0.25 \%$ trypsin without EDTA was added to each plate. After washing with PBS, $1 \times 10^{5}$ cells were collected and cultured in the dark with $5 \mu$ l Annexin V-APC and $5 \mu \mathrm{l}$ PI staining solution (Nanjing Keygen Biotech Co., Ltd.) for $15 \mathrm{~min}$ at room temperature, followed by detection using a flow cytometer (MoFloAstrios EQ; Beckman Coulter, Inc.). Annexin V-APC (+) PI (-) cells were defined as early apoptotic cells whereas Annexin V-APC (+) PI (+) cells were defined as late apoptotic cells. The flow cytometry data were analyzed using FlowJo software 8.7.1 (FlowJo LLC). The apoptotic rate was calculated as (early apoptotic cells + late apoptotic cells)/total cells $\mathrm{x} 100 \%$.

Transwell assays. HTR-8/SVneo cells were resuspended in serum-free RPMI-1640 medium and seeded into the upper chamber of the Transwell chamber $(8-\mu \mathrm{m}$ pore size; Corning, Inc.) precoated with Matrigel (BD Biosciences; Matrigel was diluted at 1:8 and added in the Transwell chamber until fully solidified at $37^{\circ} \mathrm{C}$ for $12 \mathrm{~h}$ ) in a single layer with $5 \times 10^{4}$ cells/well. RPMI-1640 medium with 10\% FBS was placed into the lower chamber. Cells were cultured for $48 \mathrm{~h}$ at $5 \% \mathrm{CO}_{2}$ at $37^{\circ} \mathrm{C}$. The cells were then fixed for 15 min with $95 \%$ ethanol at room temperature and stained for $20 \mathrm{~min}$ with $0.1 \%$ crystal violet at room temperature. Three visual fields were arbitrarily selected for each well under a light microscope (magnification, x100) to count the number of invasive cells for statistical analysis. Transwell chambers without Matrigel were used to measure cell migration in the same manner.

Matrigel-based tube formation assay. The serum-free RPMI-1640 culture medium was mixed with Matrigel at 1:1 in an ice box overnight. This mixture was then placed into 24-well plates at $150 \mu \mathrm{l} /$ well at $37^{\circ} \mathrm{C}$ for $30 \mathrm{~min}$ for solidification. HTR-8/SVneo cells were detached using trypsin, resuspended and plated into Matrigel-precoated 24-well plates at $1 \times 10^{4}$ cells/well with $500 \mu \mathrm{l}$ RPMI-1640 medium and $5 \%$ FBS. After $4 \mathrm{~h}$ incubation at $37^{\circ} \mathrm{C}$, tube formation was observed using a contrast microscope (magnification, $\mathrm{x} 100$ ). Three visual fields were arbitrarily selected per well to count the total number of bifurcation points forming $>3$ lumens.
$R T$ - $q P C R$. Total RNA was extracted from placental tissues or HTR-8/SVneo cells using TRIzol ${ }^{\circledR}$ reagent (Thermo Fisher Scientific, Inc.). The concentration and purity of the extracted RNA were determined using a UV spectrophotometer (Nano Photometer; IMPLEN GmbH). In total, $10 \mu 1$ total RNA was used for RT with 5X All-In One RT MasterMix (Applied Biological Materials, Inc.). Reverse transcription reaction conditions were as follows: $25^{\circ} \mathrm{C}$ for $10 \mathrm{~min}, 42^{\circ} \mathrm{C}$ for $15 \mathrm{~min}$ and $85^{\circ} \mathrm{C}$ for $5 \mathrm{~min}$. The cDNA was produced after the reaction and stored at $-20^{\circ} \mathrm{C}$. Using the consequent cDNA as a template, EvaGreen $2 \mathrm{X}$ qPCR MasterMix-Low Rox (cat. no. E824483-4 Shanghai Macklin Biochemical Co., Ltd.) was used for qPCR. The total reaction volume was $20 \mu \mathrm{l}$. PCR amplification conditions were as follows: Denaturation at $95^{\circ} \mathrm{C}$ for $10 \mathrm{~min}$, followed by 40 cycles of denaturation at $95^{\circ} \mathrm{C}$ for $15 \mathrm{sec}$, denaturation at $95^{\circ} \mathrm{C}$ for $1 \mathrm{~min}$, annealing/extension at $60^{\circ} \mathrm{C}$ for $30 \mathrm{sec}$ and denaturation at $60^{\circ} \mathrm{C}$ for $15 \mathrm{sec}$. The relative expression of $\mathrm{BCYRN1}$ was normalized to that of GAPDH and calculated using the $2^{-\Delta \Delta \mathrm{Cq}}$ method (28). The primer sequences were synthesized by Sangon Biotech Co., Ltd. (Table I). Each sample was performed three times independently.

Western blot analysis. Total protein was harvested from the placental tissues or HTR-8/SVneo cells using RIPA buffer (Beyotime Institute of Biotechnology), and the protein concentration was measured using a bicinchoninic acid protein quantitative kit (Sangon Biotech Co., Ltd.). A total of $50 \mu \mathrm{g}$ protein/lane was separated using $7.5 \%$ SDS-PAGE. The protein was then transferred onto nitrocellulose membranes (EMD Millipore). After blocking using $5 \%$ skim milk at room temperature for $2 \mathrm{~h}$, the membranes were probed with primary rabbit anti-human polyclonal antibodies against Wnt1 (cat. no. ab63934; 1:1,000; Abcam), $\beta$-catenin (cat. no. ab16051; 1:1,000; Abcam) and GAPDH (cat. no. ab9485; 1:1,000; Abcam) at $4^{\circ} \mathrm{C}$ overnight, followed by incubation for $2 \mathrm{~h}$ at room temperature with horseradish peroxidase-labeled goat anti-rabbit IgG secondary antibodies (cat. no. HS-GR-HRP-500; 1:10,000; Shijiazhuang No.4 Pharmaceutical Hanlin Biotechnology Co., Ltd.). Finally, enhanced chemiluminescence reagent (Immobilon Western Chemilum HRP Substrate; cat. no. WBKLS0100; EMD Millipore) was added for visualizing the membranes, before the density of bands was quantified using a Gel-Pro Analyzer 4.0 software (Media Cybernetics, Inc.). Each sample was tested three times independently.

Statistical analysis. All data were processed using SPSS 21.0 (IBM Corp.) and GraphPad Prism 6.0 (GraphPad Software, 
Table II. Comparison of baseline clinical characteristics.

\begin{tabular}{lccc}
\hline Parameter & Normal & Mild preeclampsia & Severe preeclampsia \\
\hline Number of cases, $\mathrm{n}$ & 30 & 30 & 30 \\
Age, years & $32.62 \pm 4.47$ & $31.76 \pm 5.13$ & $34.08 \pm 4.19$ \\
Gestation, weeks & $35.88 \pm 3.24$ & $35.74 \pm 3.23$ & $35.54 \pm 2.59$ \\
Body mass index at early pregnancy, $\mathrm{kg} / \mathrm{m}^{2}$ & $24.93 \pm 1.75$ & $26.48 \pm 2.03^{\mathrm{a}}$ & $25.35 \pm 2.26$ \\
Systolic blood pressure, $\mathrm{mmHg}$ & $113.50 \pm 8.71$ & $152.72 \pm 9.89^{\mathrm{b}}$ & $164.63 \pm 16.30^{\mathrm{b}, \mathrm{c}}$ \\
Diastolic blood pressure, $\mathrm{mmHg}$ & $70.97 \pm 7.03$ & $93.78 \pm 7.82^{\mathrm{b}}$ & $105.86 \pm 11.31^{\mathrm{b}, \mathrm{c}}$ \\
24 h urine protein, g & $0.207 \pm 0.087$ & $1.487 \pm 0.476^{\mathrm{b}}$ & $3.767 \pm 0.725^{\mathrm{b}, \mathrm{c}}$ \\
Fetal birth weight, $\mathrm{kg}$ & $3.318 \pm 0.352$ & $2.972 \pm 0.227^{\mathrm{b}}$ & $2.426 \pm 0.325^{\mathrm{b}, \mathrm{c}}$ \\
Neonatal asphyxia, $\mathrm{n}^{\mathrm{d}}$ & 0 & 1 & 5
\end{tabular}

${ }^{\mathrm{a}} \mathrm{P}<0.05,{ }^{\mathrm{b}} \mathrm{P}<0.01 \mathrm{vs}$. normal; ${ }^{\mathrm{c}} \mathrm{P}<0.01 \mathrm{vs}$. mild preeclampsia, one-way ANOVA analysis followed by Tukey's test. ${ }^{\mathrm{d}}$ Fisher's exact test was used to compare the number of cases of neonatal asphyxia.

Inc.). All experiments were repeated three times. The measurement data were presented as the mean \pm standard deviation and analyzed using one-way (comparison of three or more groups with only one independent variable) or two-way (comparison of three or more groups with two independent variables) analysis of variance followed by Tukey's multiple comparisons test. Pearson correlation analysis was used to analyze the correlation between two continuous variables. Categorical data were expressed as number of cases and Fisher's exact test was used to assess the association when the expected frequency was $<5$. Kendall's $\tau$-b correlation analysis was used to observe the correlation between the frequency of neonatal asphyxia and BCYRN1 expression. $\mathrm{P}<0.05$ was considered to indicate a statistically significant difference.

\section{Results}

Clinical baseline characteristics of enrolled population. There was no significant difference in age and gestational weeks in the individuals among the three groups, whilst the body mass index of the MPE group was significantly higher compared with that in the Normal group (Table II). SBP, DBP and 24-h urine protein in the MPE and SPE groups were significantly higher compared with those in the Normal group $(\mathrm{P}<0.01$; Table II). In addition, SBP, DBP and 24-h urine protein were significantly higher in the SPE group compared with in the MPE group $(\mathrm{P}<0.01$; Table II). There was no significant difference in the incidence of neonatal asphyxia among the three groups.

IncRNA BCYRN1 expression is decreased in placenta samples of patients with $P E$ and correlated with clinical features of $P E$. BCYRN1 expression in the placental tissue from the MPE and SPE groups was significantly reduced compared with in the Normal group, whilst BCYRN1 expression in the SPE group was significantly lower compared with in the MPE group (all $\mathrm{P}<0.05$; Fig. 1A). BCYRN1 expression was downregulated in the placental tissues of patients with PE, which decreased further with progressive aggravation of the disease.

The correlation and association between BCYRN1 expression and the clinical characteristics of pregnant women and newborns was analyzed further. BCYRN1 expression in placental tissues was negatively correlated with admission SBP and 24-h urine protein $(\mathrm{P}<0.05$; Fig. 1B-E). However, relative BCYRN1 expression was not correlated with neonatal birth weight or incidences of neonatal asphyxia $(\mathrm{P}>0.05)$ (data not shown).

Overexpression of IncRNA BCYRN1 promotes trophoblast cell proliferation and inhibits apoptosis. Trophoblast cells are an important component of the placental tissue, and participate in placental development and angiogenesis, where the aberrant dysfunction of trophoblast cells is closely associated with PE pathogenesis (2). HTR-8/SVneo cells were transfected with either si-BCYRN1 or pcDNA3.1-BCYRN1, following which cell viability and apoptosis of HTR-8/SVneo cells were analyzed and compared. Compared with the si-NC group, BCYRN1 expression was significantly decreased in the si-BCYRN1 group, whereas BCYRN1 expression in the pcDNA3.1-BCYRN1 group was significantly elevated compared with that in the pcDNA3.1-NC group (all $\mathrm{P}<0.05$; Fig. 2A), suggesting that the transfection was effective. Compared with the si-NC group, the viability of trophoblast cells was reduced in the si-BCYRN1 group, but was increased at $72 \mathrm{~h}$ in the pcDNA3.1-BCYRN1 group compared with the pcDNA3.1 group (all $\mathrm{P}<0.05$; Fig. 2B). Similarly, the apoptosis rate of the si-BCYRN1 group was also significantly elevated compared with the si-NC group, whilst that of the pcDNA3.1-BCYRN1 group was significantly decreased compared with the pcDNA3.1 group ( $\mathrm{P}<0.05$; Fig. 2C).

Overexpression of IncRNA BCYRN1 promotes trophoblast cell migration and invasion. The migration, invasion and tube-forming abilities of trophoblast cells are crucial to the establishment of maternal-fetal circulation (29). Reduced invasive ability of trophoblast cells and subsequent abnormal remodeling of the spiral artery are important features of PE (30). The number of migratory HTR-8/SVneo cells in the si-BCYRN1 group was significantly decreased compared with that in the si-NC group, whilst the number of migratory cells in the pcDNA3.1-BCYRN1 group was significantly increased compared with the pcDNA3.1 group (all $\mathrm{P}<0.05$; Fig. 3A). 
A

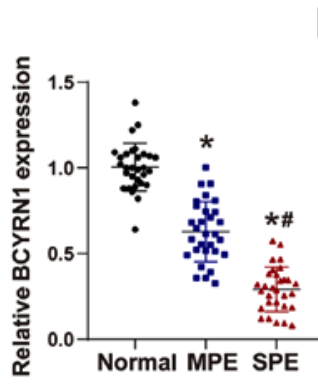

B

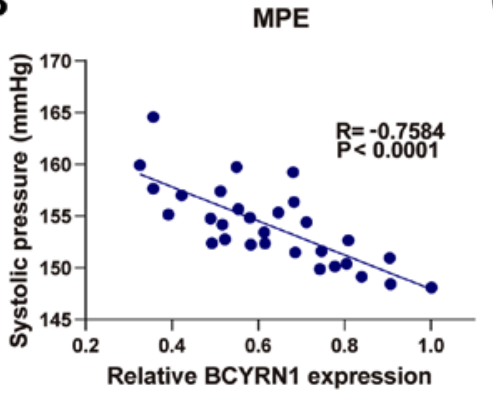

C

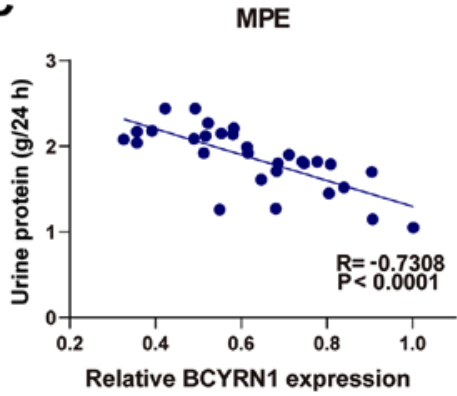

D

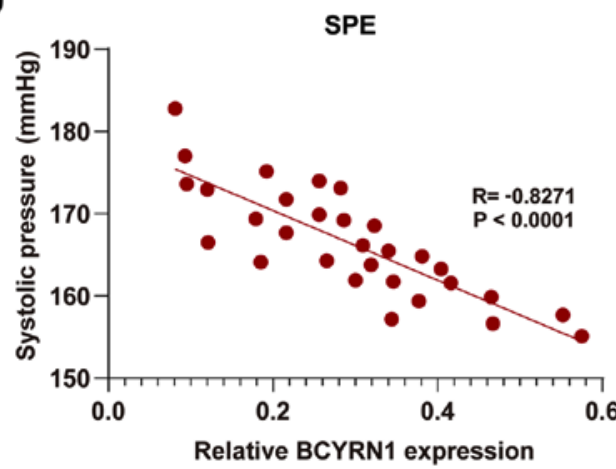

E

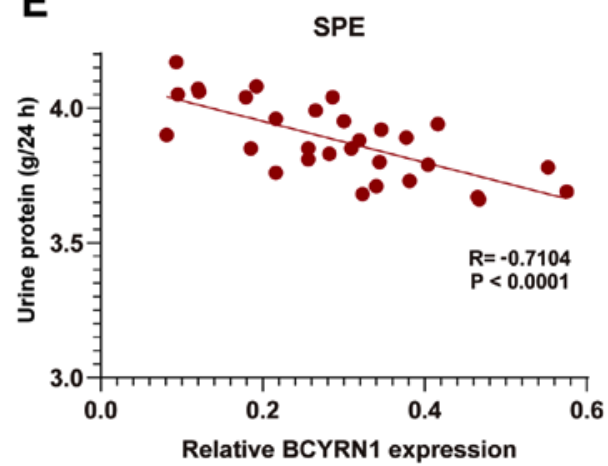

Figure 1. Long non-coding RNA BCYRN1 expression is reduced in placenta of patients with PE and is correlated with the clinical parameters of PE. (A) Expression of BCYRN1 in placental tissue was measured by reverse transcription-quantitative PCR. Each sample was tested three times independently. Expression of BCYRN1 in placental tissues from the MPE group was negatively correlated with (B) systolic blood pressure and (C) 24-h urine protein. Expression of BCYRN1 in placenta tissues from the SPE group was negatively correlated with (D) systolic blood pressure and (E) 24-h urine protein relevant. N=30. One-way ANOVA followed by Tukey's multiple comparisons test was used for panel A. Pearson's correlation analysis was used for panels B-E. ${ }^{*} \mathrm{P}<0.05$ vs. Normal group; ${ }^{\text {P }}<0.05$ vs. MPE. BCYRN1, brain cytoplasmic RNA 1; PE, preeclampsia; MPE, mild PE; SPE, severe PE.

Compared with the si-NC group, the number of invasive cells was significantly decreased in the si-BCYRN1group, but significantly increased in the pcDNA3.1-BCYRN1 group compared with the pcDNA3.1 group (all $\mathrm{P}<0.05$; Fig. $3 \mathrm{~B}$ ). Compared with the si-NC group, the number of bifurcation points in the si-BCYRN1 group was significantly decreased, whilst that of the pcDNA3.1-BCYRN1 group was significantly enhanced compared with the pcDNA3.1 group (all $\mathrm{P}<0.05$; Fig. 3C).

IncRNA BCYRN1 activates the Wnt/ $\beta$-catenin axis in trophoblast cells. The $\mathrm{Wnt} / \beta$-catenin pathway can regulate the proliferation and apoptosis of trophoblast cells, and is pivotal for trophoblast cell invasion and implantation into the endometrium (31). It was hypothesized that lncRNA BCYRN1 may regulate the $\mathrm{Wnt} / \beta$-catenin signaling axis in trophoblast cells. The levels of Wnt1 and $\beta$-catenin in the placental tissues from patients with SPE were significantly lower compared with those in the Normal group and patients with MPE (all $\mathrm{P}<0.05$; Fig. 4A). Furthermore, the levels of Wnt1 and $\beta$-catenin were significantly reduced in the si-BCYRN1 group compared with those in the si-NC group, but were elevated in the pcDNA3.1-BCYRN1 group compared with in the pcDNA3.1 group (all $\mathrm{P}<0.05$; Fig. 4B). This suggests that lncRNA BCYRN1 can promote the activation of the Wnt/ $\beta$-catenin axis in trophoblast cells.

lncRNA BCYRN1 promotes trophoblast proliferation and blocks apoptosis by activating the Wnt/ $\beta$-catenin axis. To investigate whether IncRNA BCYRN1 affects the viability and apoptosis of trophoblast cells by activating the Wnt/ $\beta$-catenin pathway, the Wnt pathway inhibitor XAV939 or DMSO was applied to treat HTR8/SVneo cells overexpressing BCYRN1. Compared with the pcDNA3.1-BCYRN1 group, the viability of the pcDNA3.1-BCYRN1 + XAV939 group was significantly reduced at 72 and $96 \mathrm{~h}(\mathrm{P}<0.05$; Fig. 5A), whilst the apoptosis rate was enhanced $(\mathrm{P}<0.05$; Fig. $5 \mathrm{~B})$. These results suggested that XAV939 can attenuate the effect of BCYRN1 overexpression on HTR8/SVneo cell viability and apoptosis.

\section{Discussion}

Early onset of PE has been reported to be the result of poor placental implantation, leading to an inflammatory cascade resulting in high blood pressure (32). To the best of our knowledge, the present study was the first to determine the expression profile of lncRNA BCYRN1 in placental tissues with $\mathrm{PE}$ and to explore the potential regulatory effects of BCYRN1 on the physiology of trophoblast cells. The expression level of BCYRN1 was found to be downregulated in placenta tissues with $\mathrm{PE}$; a similar reduction in trophoblast cells also suppressed the migration and invasion of trophoblasts. BCYRN1 may therefore be involved in the development of placental abnormalities during the early stages of PE. These results suggested that the pathogenesis of PE may be associated with epigenetic changes.

The present study demonstrated that BCYRN1 expression was reduced in the placental tissue from patients with PE. Additionally, BCYRN1 expression in placental tissues from patients with MPE was lower compared with that of SPE. This 
A

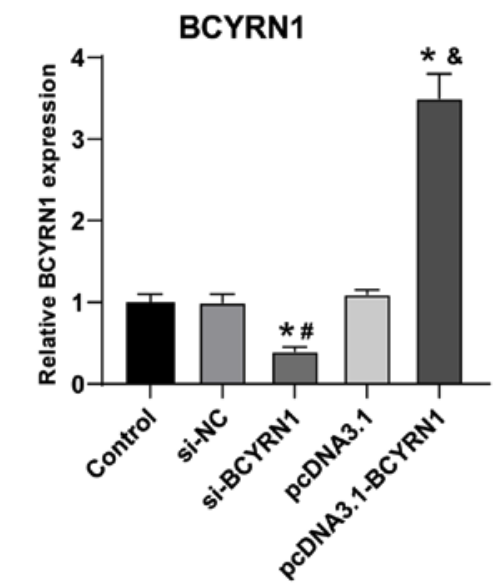

C

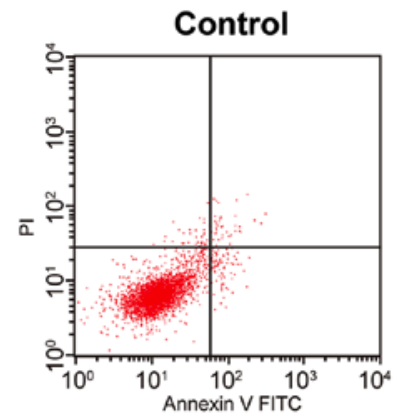

si-BCYRN1

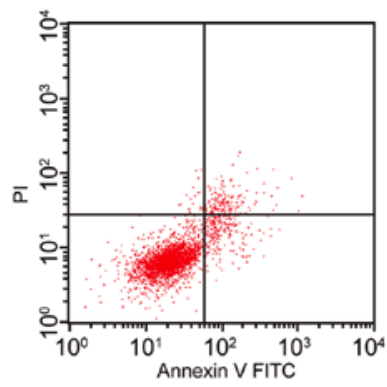

pcDNA3.1-BCYRN1

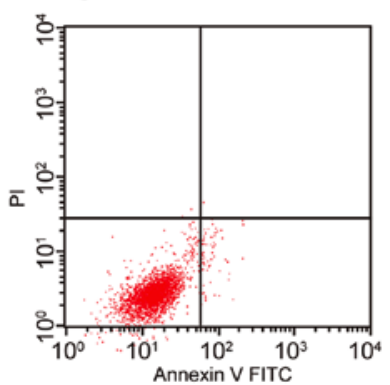

B
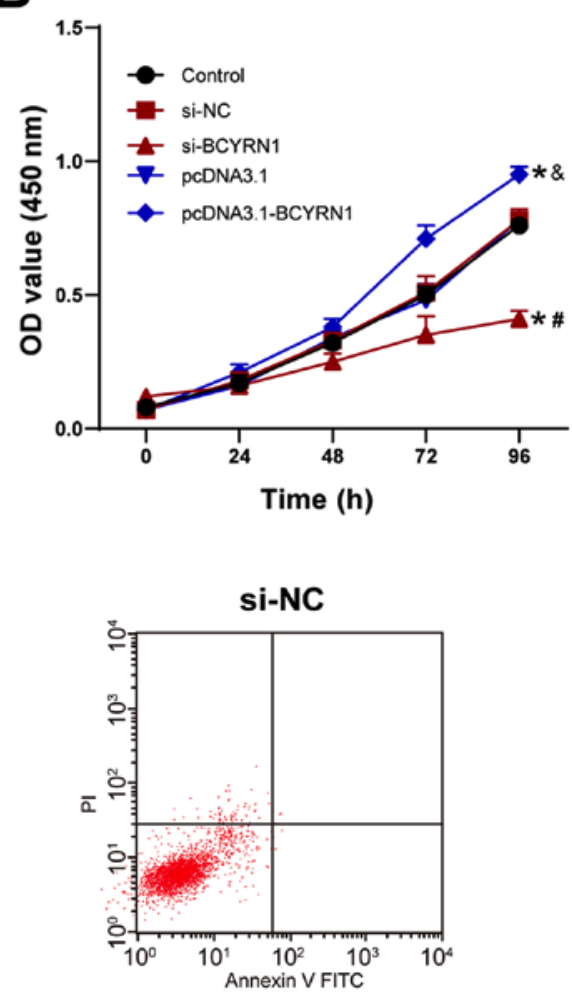

pcDNA3.1
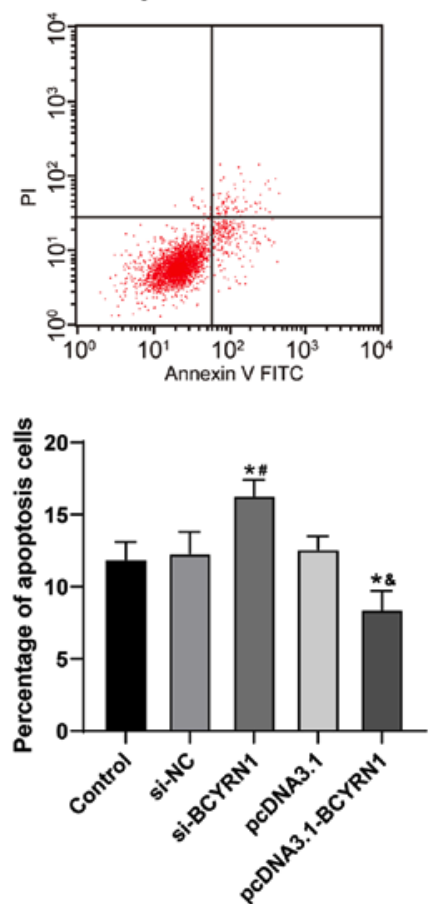

Figure 2. Overexpression of long non-coding RNA BCYRN1 promotes trophoblast cell viability and inhibits apoptosis. (A) After $48 \mathrm{~h}$ transfection, BCYRN1 expression was detected by reverse transcription-quantitative PCR. (B) Cell viability was detected by Cell Counting Kit- 8 assay. (C) Apoptosis rate was detected by flow cytometry. Cell experiments were performed three times. The data are expressed as the mean \pm standard deviation. Data in panels A and C were analyzed by one-way ANOVA, and data in panel B were analyzed by two-way ANOVA, followed by Tukey's multiple comparisons test. "P $<0.05$ vs. control group; ${ }^{\#} \mathrm{P}<0.05$ vs. si-NC group; ${ }^{\circledR} \mathrm{P}<0.05$ vs. pcDNA3.1 group. BCYRN1, brain cytoplasmic RNA 1; PE, preeclampsia; NC, negative control, si, small interfering; OD, optical density; PI, propidium iodide.

suggested that BCYRN1 expression is downregulated in the placental tissues of patients with $\mathrm{PE}$, and is decreased further as the disease worsens, suggesting that BCYRN1 may be associated with the severity of PE, which may have clinical relevance. Importantly, BCYRN1 expression in placental tissues was negatively correlated with admission SBP and 24-h urine protein levels. The incidence of pregnancy adverse effects increases in patients with elevated 24 -h proteinuria (33). 

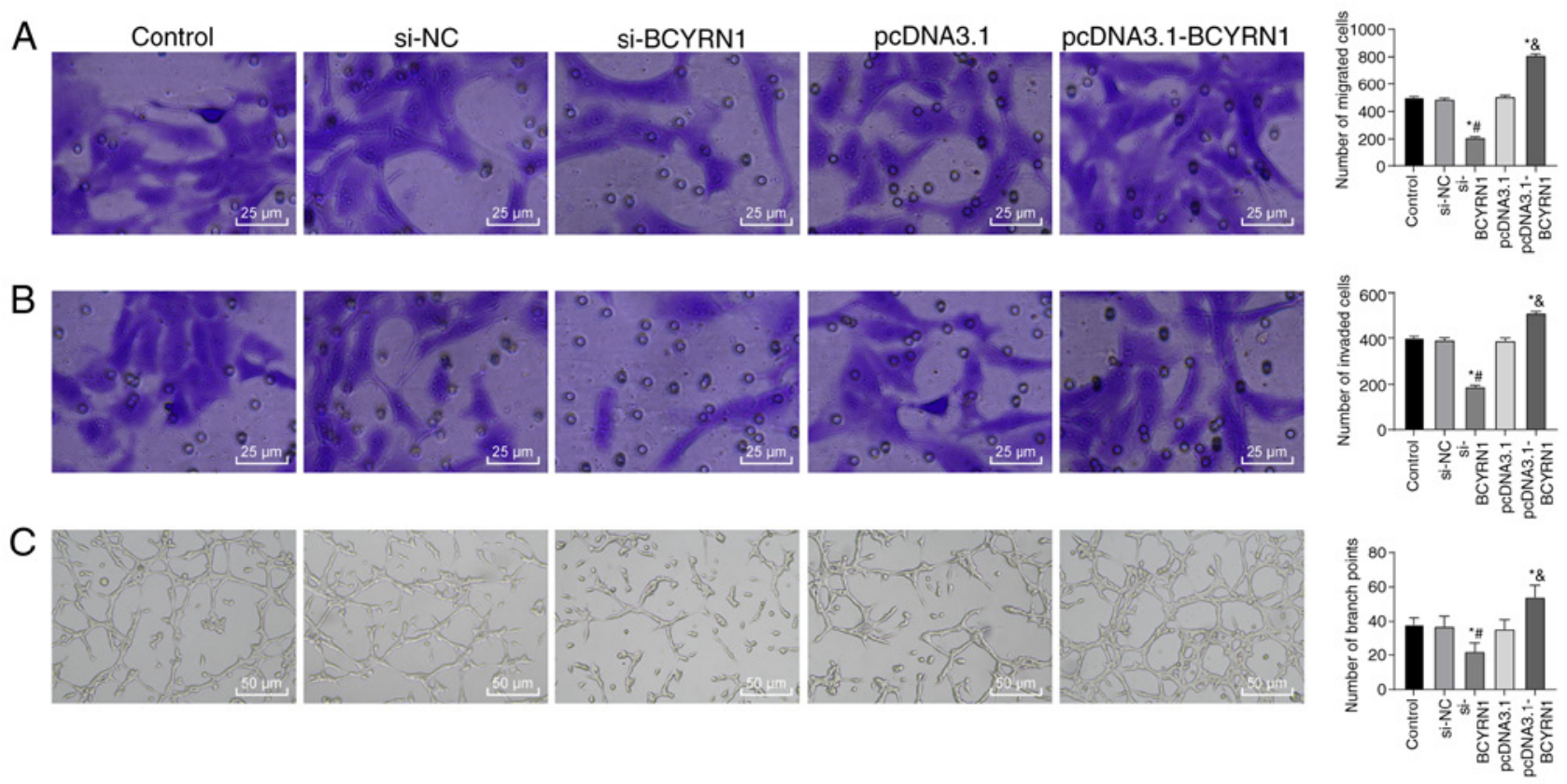

Figure 3. Overexpression of long non-coding RNA BCYRN1 promotes migration, invasion and tube formation of trophoblast cells. After transfection with si-BCYRN1 and pcDNA3.1-BCYRN1, the migratory and invasive ability of HTR-8/SVneo cells was measured by (A) Transwell migration assay and (B) Transwell invasion assay. Scale bars, $25 \mu \mathrm{m}$. (C) Tube forming ability was detected by cell tube-forming test. Scale bars, $50 \mu \mathrm{m}$. All experiments were repeated three times and the data are expressed as the mean \pm standard deviation. Data were analyzed by one-way ANOVA followed by Tukey's multiple comparisons test. ${ }^{*} \mathrm{P}<0.05$ vs. Control; " $\mathrm{P}<0.05$ vs. si-NC; ${ }^{\circledR} \mathrm{P}<0.05$ vs. pcDNA3.1. BCYRN1, brain cytoplasmic RNA 1; PE, preeclampsia; NC, negative control, si, small interfering.
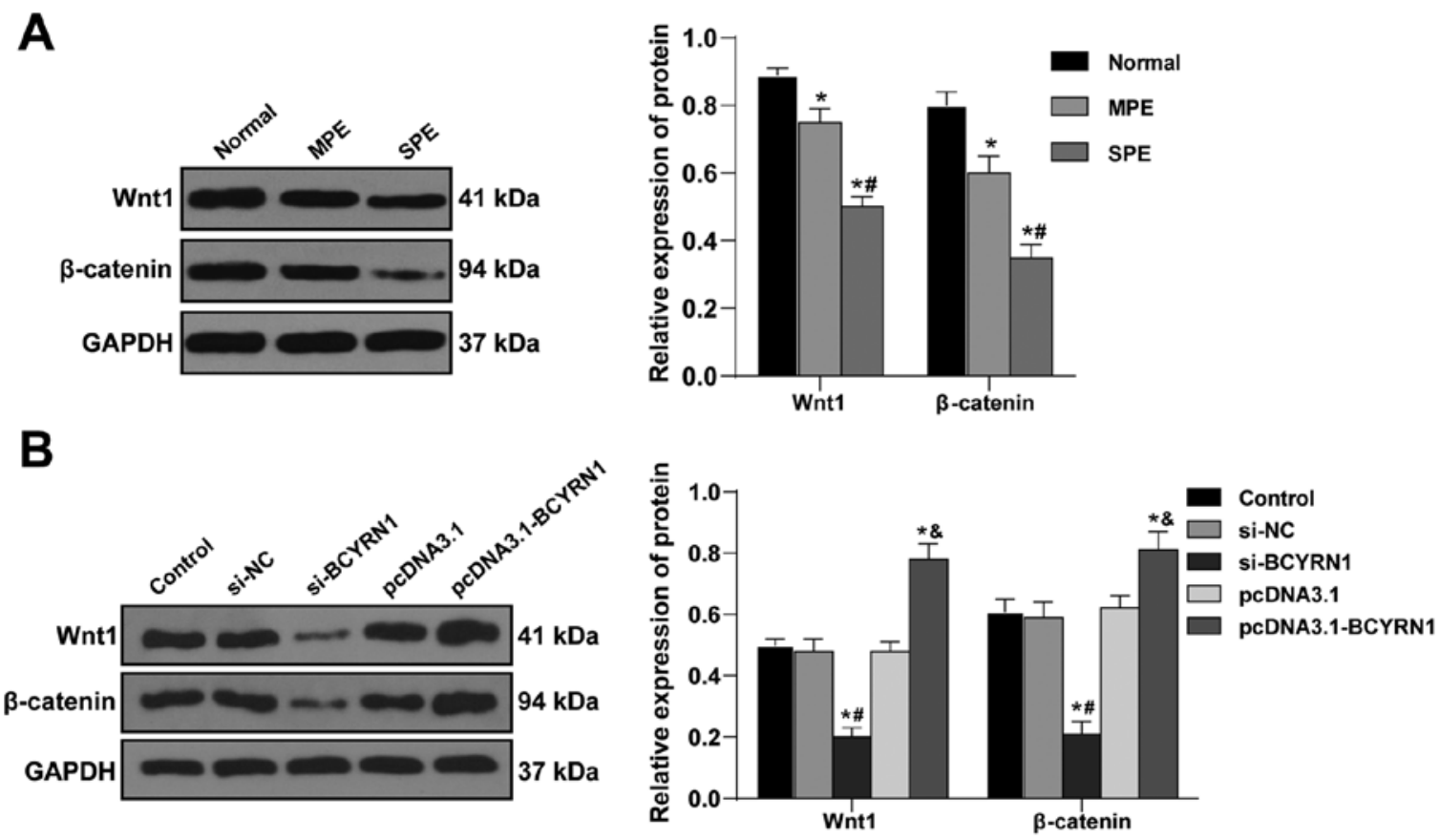

Figure 4. Long non-coding RNA BCYRN1 activates the Wnt/ $\beta$-catenin pathway in trophoblast cells. (A) Expressions of Wnt1 and $\beta$-catenin in placenta tissues from normal pregnant women and patients with $\mathrm{PE}$ were measured via western blotting. $\mathrm{P}<0.05$ vs. Normal group; ${ }^{\text {}} \mathrm{P}<0.05$ vs. MPE group; Three samples were tested independently in each group. (B) Expression of Wnt1 and $\beta$-catenin in HTR8/SVneo cells of each group was measured via western blotting. ${ }^{*} \mathrm{P}<0.05$ vs. Control; ${ }^{\prime} \mathrm{P}<0.05$ vs. si-NC; ${ }^{\&} \mathrm{P}<0.05$ vs. pcDNA3.1. The cell experiments were conducted three times independently. One-way ANOVA analysis was used for comparison between groups, followed by Tukey's multiple comparisons test. BCYRN1, brain cytoplasmic RNA 1; PE, preeclampsia; MPE, mild PE; SPE, severe PE; NC, negative control, si, small interfering.

These results provided epidemiological evidence that reduced expression of lncRNA BCYRN1 is correlated with increased adverse pregnancy effects. However, the sample size in the present study was small, and multi-center and large-scale experiments are required to verify this finding. Additionally, the biological sample used in the present study was placental tissues after delivery. In future studies, the expression of BCYRN1 in maternal serum should also be measured. 

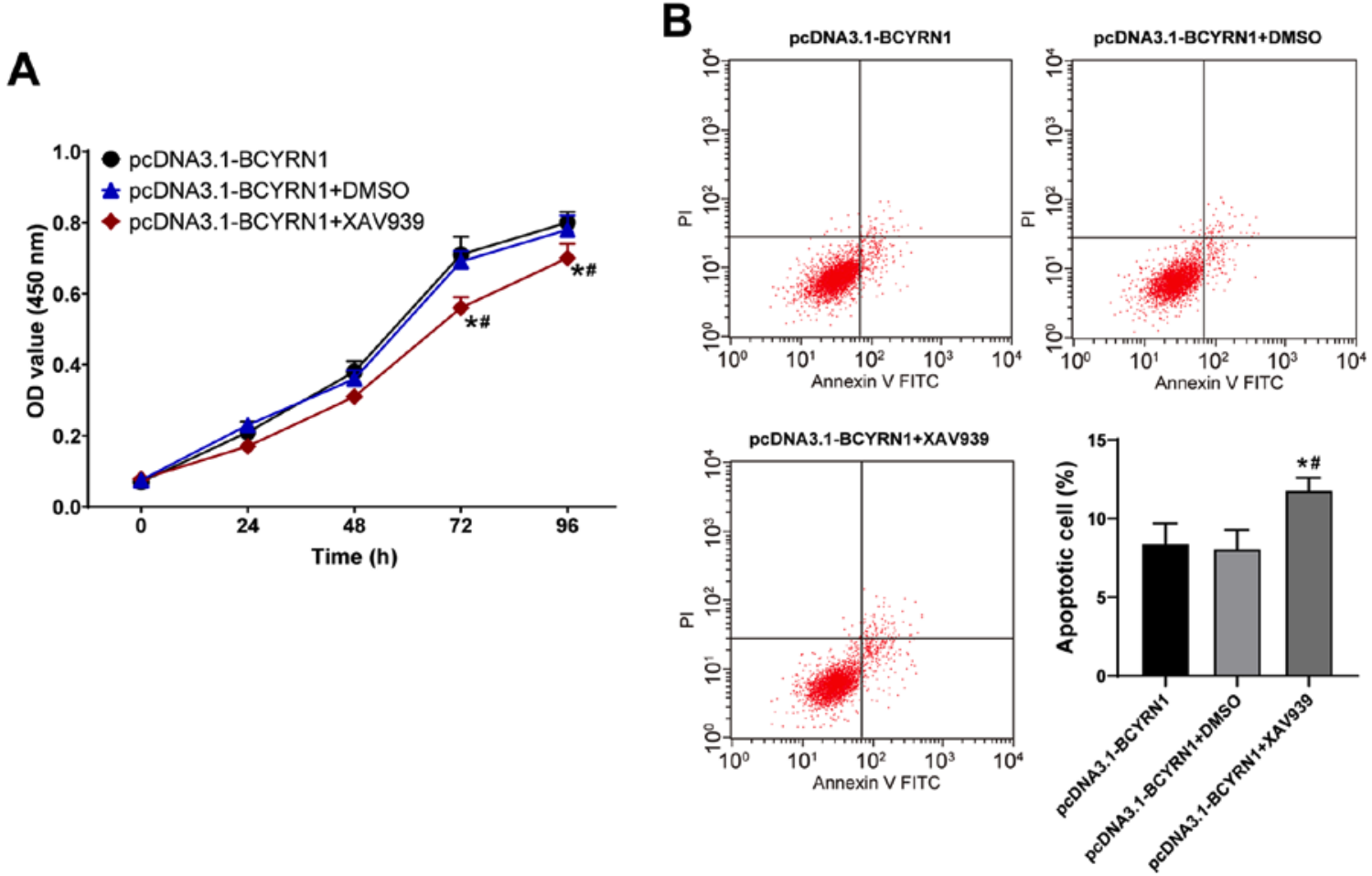

Figure 5. XAV939 can attenuate the effects of BCYRN1 overexpression on the viability and apoptosis of HTR8/SVneo cells. HTR8/SVneo cells overexpressing BCYRN1 were treated with either the Wnt inhibitor XAV939 or DMSO. (A) Cell viability was measured using Cell Counting Kit-8. (B) Apoptosis was detected via flow cytometry. Cell experiments were repeated three times and the data are expressed as the mean \pm standard deviation. Data in panel A were analyzed by two-way ANOVA, whilst data in panel B were analyzed by one-way ANOVA followed by Tukey's multiple comparisons test. "P<0.05 vs. pcDNA3.1-BCYRN1; ${ }^{\text {"P}} \mathrm{P}<0.05$ vs. pcDNA3.1-BCYRN1 + DMSO. BCYRN1, brain cytoplasmic RNA 1; OD, optical density; PI, propidium iodide.

At present, the clinical consensus is that PE is caused by poor placental formation during early pregnancy (2). During placental implantation under PE, trophoblast cells lack invasiveness, which leads to the incomplete remodeling of the spiral artery, thereby causing placental ischemia and hypoxia, oxidative stress and systemic inflammatory responses (2). The present study focused on the biological behavior of trophoblast cells, namely trophoblast migration and invasion, which is an early pathological process in placental dysplasia (2). HTR-8/SVneo cells have been used extensively to study the biological activity of trophoblasts in vitro (26). Therefore, HTR-8/SVneo cells were used in the present study to estimate the in vitro role of BCYRN1 in PE. After transfection of HTR-8/SVneo cells, the viability, migratory, invasive and tube-forming capabilities of trophoblast cells in the si-BCYRN1 group were impaired, whilst the opposite effects were observed in the pcDNA3.1-BCYRN1 group. The migration, invasion and tube formation by trophoblasts into the endometrium and vascular system are key steps during placenta formation, in addition to contributing to the pathogenesis of PE $(24,29,34,35)$. The present study revealed that the apoptosis rate in the si-BCYRN1 group was higher, whilst that in the pcDNA3.1-BCYRN1 group was decreased. Decreased trophoblast proliferation and invasion, and increased apoptosis constitute the main underlying causes of PE $(30,36)$. These previous findings supported the data from the present study that BCYRN1 overexpression increased trophoblast cell proliferation, migration, invasion and tube-forming abilities whilst blocking apoptosis to alleviate PE progression. However, the effect of BCYRN1 on the expression of enzymes in trophoblast cells, the effect of BCYRN1 on ischemia, hypoxia and inflammatory factors in placental tissues and potential targets for the treatment of PE all require further study.

Early in pregnancy, enhanced $\mathrm{Wnt} / \beta$-catenin signaling is a prerequisite for the proper implantation and invasion of trophoblast cells (37). The Wnt/ $\beta$-catenin axis regulates the apoptosis and invasion of trophoblast cells and is essential for placentation (32). It was therefore speculated in the present study that lncRNA BCYRN1 may regulate the $\mathrm{Wnt} / \beta$-catenin pathway in trophoblast cells. The levels of Wnt1 and $\beta$-catenin proteins in the si-BCYRN1 and the pcDNA3.1-BCYRN1 groups were decreased and elevated, respectively. To verify if lncRNA BCYRN1 exerts the biological effects on trophoblast cells by evoking the Wnt/ $\beta$-catenin axis, the Wnt pathway inhibitor XAV939 was used to treat HTR8/SVneo cells overexpressing BCYRN1. Compared with that in the pcDNA3.1-BCYRN1 group, cell viability in the pcDNA3.1-BCYRN1 + XAV939 group was inhibited, whilst the apoptosis rate was enhanced. Consistently, downregulated activity of the Wnt pathway may result in trophoblast dysfunction, which can contribute to the pathogenesis of PE (38). Results from the present study suggested that IncRNA BCYRN1 can promote the activation of the Wnt/ $\beta$-catenin axis in trophoblast cells.

In conclusion, the present study indicated that BCYRN1 increased trophoblast viability and prevented apoptosis by activating the Wnt/ $/$-catenin pathway to potentially delay or block the onset of PE. The expression pattern of IncRNA BCYRN1 in placental tissues from patients with $\mathrm{PE}$ was detected to explore the regulatory mechanism of IncRNA BCYRN1 on the biological behavior of trophoblasts. In the present study, the classical Wnt pathway was found to regulate cell behavior downstream 
of BCYRN1, though the non- $\beta$-catenin-dependent Wnt pathway has not been studied. In addition, whether BCYRN1 participates in PE processes in other ways, such as by absorbing microRNA sponging, requires further study. Further studies will be performed to determine the role of BCYRN1 in the biological function of trophoblast cells from the perspective of epigenetics and transcriptomics.

\section{Acknowledgements}

Not applicable.

\section{Funding}

This work was funded by the Research Center for the Development of Primary Health Care in Sichuan Province in 2019, North Sichuan Medical College (grant no. SWFZ19-Y-35), the 'Sichuan Grassroots Health and Health Development Research Team', a high-level Social Science Research Team of Sichuan Province [Sichuan Social Union Issued (grant no. 2017 No. 43)], the 2019 Municipal School-School Cooperative Scientific Research Project in Nanchong (North Sichuan Medical College; grant nos. 19SXHZ0291 and 19SXHZ0435) and Nanchong Municipal Applied Technology Research and Development Fund Project in 2020 (grant no. 20YFZJ0099).

\section{Availability of data and materials}

The datasets used and/or analyzed during the current study are available from the corresponding author on reasonable request.

\section{Authors' contributions}

LC designed the study and performed the experiments. LC, QS and BF collected the data. LC, QS and YC analyzed the data. LC, BF prepared the manuscript. LC and YC confirm the authenticity of all the raw data. All authors read and approved the final manuscript.

\section{Ethics approval and consent to participate}

The present study was approved by the Academic Ethics Committee of the Affiliated Hospital of North Sichuan Medical College (Nanchong, China) and informed consent was obtained from all individuals.

\section{Patient consent for publication}

Not applicable.

\section{Competing interests}

The authors declare that they have no competing interests.

\section{References}

1. Dymara-Konopka W, Laskowska M and Oleszczuk J: Preeclampsia-current management and future approach. Curr Pharm Biotechnol 19: 786-796, 2018.
2. Rana S, Lemoine E, Granger JP and Karumanchi SA: Preeclampsia: Pathophysiology, challenges, and perspectives. Circ Res 124: 1094-1112, 2019.

3. Malik A, Jee B and Gupta SK: Preeclampsia: Disease biology and burden, its management strategies with reference to India Pregnancy Hypertens 15: 23-31, 2019.

4. Suvakov S, Bonner E, Nikolic V, Jerotic D, Simic TP, Garovic VD, Lopez-Campos G and Mcclements L: Overlapping pathogenic signalling pathways and biomarkers in preeclampsia and cardiovascular disease. Pregnancy Hypertens 20: 131-136, 2020.

5. Cheng SB and Sharma S: Preeclampsia and health risks later in life: An immunological link. Semin Immunopathol 38: 699-708, 2016.

6. Chen Q, Jiang S, Liu H, Gao Y, Yang X, Ren Z, Gao Y, Xiao L, $\mathrm{Hu} \mathrm{H}, \mathrm{Yu} \mathrm{Y}$, et al: Association of lncRNA SH3PXD2A-AS1 with preeclampsia and its function in invasion and migration of placental trophoblast cells. Cell Death Dis 11: 583, 2020.

7. Li Q, Wang T, Huang S, Zuo Q, Jiang Z, Yang N and Sun L: lncRNA MALAT1 affects the migration and invasion of trophoblast cells by regulating FOS expression in early-onset preeclampsia. Pregnancy Hypertens 21: 50-57, 2020.

8. Apicella C, Ruano CSM, Mehats C, Miralles F and Vaiman D The role of epigenetics in placental development and the etiology of preeclampsia. Int J Mol Sci 20: 2837, 2019.

9. Mcaninch D, Roberts CT and Bianco-Miotto T: Mechanistic insight into long noncoding RNAs and the placenta. Int J Mol Sci 18: 1371, 2017.

10. He X, He Y, Xi B, Zheng J, Zeng X, Cai Q, Ouyang Y, Wang C, Zhou X, Huang $\mathrm{H}$, et al: lncRNAs expression in preeclampsia placenta reveals the potential role of lncRNAs contributing to preeclampsia pathogenesis. PLoS One 8: e81437, 2013.

11. Long W, Rui C, Song X, Dai X, Xue X, Lu Y, Shen R, Li J, Li J and Ding H: Distinct expression profiles of lncRNAs between early-onset preeclampsia and preterm controls. Clin Chim Acta 463: 193-199, 2016.

12. Harati-Sadegh M, Kohan L, Teimoori B, Mehrabani M and Salimi S: The effects of placental long noncoding RNA H19 polymorphisms and promoter methylation on $\mathrm{H} 19$ expression in association with preeclampsia susceptibility. IUBMB Life 72 : 413-425, 2020

13. Mohammadpour-Gharehbagh A, Jahantigh D, Saravani M, Harati-Sadegh M, Maruie-Milan R, Teimoori B and Salimi S: Impact of HOTAIR variants on preeclampsia susceptibility based on blood and placenta and in silico analysis. IUBMB Life 71: 1367-1381, 2019.

14. Wu HY, Wang XH, Liu K and Zhang JL: IncRNA MALAT1 regulates trophoblast cells migration and invasion via miR-206/IGF-1 axis. Cell Cycle 19: 39-52, 2020.

15. Yang X and Meng T: Long noncoding RNA in Preeclampsia: Transcriptional noise or innovative indicators? Biomed Res Int 2019: 5437621, 2019.

16. Sosinska-Zawierucha P, Zawierucha P, Breborowicz A and Barciszewski J: Prediction of secondary and tertiary structures of human BC200 RNA (BCYRN1) based on experimental and bioinformatic cross-validation. Biochem J 475: 2727-2748, 2018.

17. Huang W, Zhou R, Mao L, Deng C and Dang X: Esophageal cancer related gene-4 inhibits the migration and proliferation of oral squamous cell carcinoma through BC200 lncRNA/MMP-9 and -13 signaling pathway. Cell Signal 62: 109327, 2019.

18. Wu K, Xu K, Liu K, Huang J, Chen J, Zhang J and Zhang N: Long noncoding RNA BC200 regulates cell growth and invasion in colon cancer. Int J Biochem Cell Biol 99: 219-225, 2018.

19. Zhang XY, Tang XY, Ma LJ, Guo YL, Li XS, Zhao LM, Tian CJ, Cheng DJ, Chen ZC and Zhang LX: Schisandrin B down-regulated lncRNA BCYRN1 expression of airway smooth muscle cells by improving miR-150 expression to inhibit the proliferation and migration of ASMC in asthmatic rats. Cell Prolif 50: e12382, 2017.

20. Zhang XY, Zhang LX, Tian CJ, Tang XY, Zhao LM, Guo YL, Cheng DJ, Chen XL, Ma LJ and Chen ZC: lncRNAs BCYRN1 promoted the proliferation and migration of rat airway smooth muscle cells in asthma via upregulating the expression of transient receptor potential 1. Am J Transl Res 8: 3409-3418, 2016.

21. Xie D, Zhu J, Liu Q, Li J, Song M, Wang K, Zhou Q, Jia Y and Li T: Dysregulation of HDAC9 represses trophoblast cell migration and invasion through TIMP3 activation in Preeclampsia. Am J Hypertens 32: 515-523, 2019.

22. Zou AX, Chen B, Li QX and Liang YC: MiR-134 inhibits infiltration of trophoblast cells in placenta of patients with preeclampsia by decreasing ITGB1 expression. Eur Rev Med Pharmacol Sci 22: 2199-2206, 2018. 
23. Hannon T, Innes BA, Lash GE, Bulmer JN and Robson SC: Effects of local decidua on trophoblast invasion and spiral artery remodeling in focal placenta creta-an immunohistochemical study. Placenta 33: 998-1004, 2012.

24. Nawrocki B, Polette M, Marchand V, Maquoi E, Beorchia A, Tournier JM, Foidart JM and Birembaut P: Membrane-type matrix metalloproteinase-1 expression at the site of human placentation. Placenta 17: 565-572, 1996.

25. Perl M, Lomas-Neira J, Venet F, Chung CS and Ayala A: Pathogenesis of indirect (secondary) acute lung injury. Expert Rev Respir Med 5: 115-126, 2011.

26. Graham CH, Hawley TS, Hawley RG, Macdougall JR, Kerbel RS Khoo N and Lala PK: Establishment and characterization of first trimester human trophoblast cells with extended lifespan. Exp Cell Res 206: 204-211, 1993.

27. Abou-Kheir W, Barrak J, Hadadeh O and Daoud G: HTR-8/SVneo cell line contains a mixed population of cells. Placenta 50: 1-7, 2017.

28. Livak KJ and Schmittgen TD: Analysis of relative gene expression data using real-time quantitative PCR and the 2(-Delta Delta C(T)) Method. Methods 25: 402-408, 2001.

29. Ji L, Brkic J, Liu M, Fu G, Peng C and Wang YL: Placental trophoblast cell differentiation: Physiological regulation and pathological relevance to preeclampsia. Mol Aspects Med 34: 981-1023, 2013.

30. Alasztics B, Kukor Z, Panczel Z and Valent S: The pathophysiology of preeclampsia in view of the two-stage model. Ory Hetil 153: 1167-1176, 2012 (In Hu).

31. Zhang Z, Wang X,Zhang L, Shi Y, Wang J and Yan H: Wnt/ $\beta$-catenin signaling pathway in trophoblasts and abnormal activation in preeclampsia (Review). Mol Med Rep 16: 1007-1013, 2017.

32. Ogge G, Chaiworapongsa T, Romero R, Hussein Y, Kusanovic JP, Yeo L, Kim CJ and Hassan SS: Placental lesions associated with maternal underperfusion are more frequent in early-onset than in late-onset preeclampsia. J Perinat Med 39: 641-652, 2011.
33. Bouzari Z, Javadiankutenai M, Darzi A and Barat S: Does proteinura in preeclampsia have enough value to predict pregnancy outcome? Clin Exp Obstet Gynecol 41: 163-168, 2014.

34. Kokkinos MI, Murthi P, Wafai R, Thompson EW and Newgreen DF: Cadherins in the human placenta-epithelial-mesenchymal transition (EMT) and placental development. Placenta 31: 747-755, 2010.

35. Zhang Y, Cao L, Jia J, Ye L, Wang Y, Zhou B and Zhou R: CircHIPK 3 is decreased in preeclampsia and affects migration, invasion, proliferation, and tube formation of human trophoblast cells. Placenta 85: 1-8, 2019.

36. Fu G, Ye G, Nadeem L, Ji L, Manchanda T, Wang Y, Zhao Y, Qiao J, Wang YL, Lye S, et al: MicroRNA-376c impairs transforming growth factor- $\beta$ and nodal signaling to promote trophoblast cell proliferation and invasion. Hypertension 61: 864-872, 2013.

37. Van Der Horst PH, Wang Y, Van Der Zee M, Burger CW and BlokLJ: Interaction between sex hormones and WNT/beta-catenin signal transduction in endometrial physiology and disease. Mol Cell Endocrinol 358: 176-184, 2012.

38. Zhang Z, Li H, Zhang L, Jia L and Wang P: Differential expression of $\beta$-catenin and Dickkopf- 1 in the third trimester placentas from normal and preeclamptic pregnancies: A comparative study. Reprod Biol Endocrinol 11: 17, 2013.

This work is licensed under a Creative Commons Attribution-NonCommercial-NoDerivatives 4.0 International (CC BY-NC-ND 4.0) License. 